\title{
A method for single nucleotide polymorphism selection for parentage assessment in goats ${ }^{1}$
}

\author{
A. Talenti, ${ }^{* 2}$ E. L. Nicolazzi,† S. Chessa,ł S. Frattini, ${ }^{*}$ R. Moretti,ł B. Coizet, ${ }^{*}$ L. Nicoloso, ${ }^{*}$ L. Colli,§ \\ G. Pagnacco, ${ }^{*}$ A. Stella,†‡ P. Ajmone-Marsan,§ G. Ptak,\#ll and P. Crepaldi* \\ *Dipartimento di Scienze Veterinarie e Sanità Pubblica, Università di Milano, via Celoria 10, Milano, 20133, Italy \\ †Fondazione Parco Tecnologico Padano (PTP), Via Einstein, Cascina Codazza, Lodi, 26900, Italy \\ †lstituto di Biologia e Biotecnologia Agraria (IBBA-CNR), Consiglio Nazionale delle Ricerche, Via Einstein, Cascina Codazza, Lodi, 26900, Italy \\ §Istituto di Zootecnica, Università Cattolica del Sacro Cuore Via Emilia Parmense, Piacenza, 29122, Italy \\ \#Dipartimento di Scienze Biomediche Comparate, Università di Teramo, Piazza Aldo Moro 45, Teramo, 64100, Italy \\ IIInstitute of Animal Production, ul. Sarego 2, Krakow 31-047, Poland
}

\begin{abstract}
Accurate pedigrees are essential to optimize genetic improvement and conservation of animal genetic resources. In goats, the use of mating groups and kidding management procedures hamper the identification of parentage. Small panels of single nucleotide polymorphisms (SNP) have been proposed in other species to substitute microsatellites for parentage assessment. Using data from the current GoatSNP50 chip, we developed a new 3-step procedure to identify a low-density SNP panel for highly accurate parentage assessment. Methodologies for SNP selection used in other species are less suitable in the goat because of uncertainties in the genome assembly. The procedure developed in this study is based on parent-offspring identification and on estimation of Mendelian errors, followed by canonical discriminant analysis identification and stepwise regression reduction. Starting from a reference sample of 109 Alpine goats with known pedigree relationships, we first identified a panel of 200 SNP that was further reduced to 2 final panels of 130 and 114 SNP with random coincidental match inclusion of $1.51 \times 10^{-57}$ and $2.94 \times$ $10^{-34}$, respectively. In our reference data set, all panels correctly identified all parent-offspring combinations, revealing a $40 \%$ pedigree error rate in the information provided by breeders. All reference trios were confirmed by official tests based on microsatellites. Panels were also tested on Saanen and Teramana breeds. Although the testing on a larger set of breeds in the reference population is still needed to validate these results, our findings suggest that our procedure could identify SNP panels for accurate parentage assessment in goats or in other species with unreliable marker positioning.
\end{abstract}

Received July 7, 2015.

Accepted January 25, 2016.

${ }^{1}$ The authors declare they have no competing interests.

${ }^{2}$ Corresponding author: andrea.talenti@unimi.it
Key words: parentage analysis, goat, single nucleotide polymorphism

\section{INTRODUCTION}

Accurate assessment of relationships between individuals in a population is one of the main requirements for a successful genetic improvement program. In goats, the accurate registration of parentage is often hampered by some widespread management practices, such as the use of mating groups, summer pasturing, and collective nurseries for kids at early stages of life. These practices usually lead to high rates of pedigree registration errors, which may involve one or both parents. Consequently, genetic progress slows down.

The use of molecular markers to determine parentage has been extensively studied in livestock (Heaton et al., 2002; Werner et al., 2004; Fisher et al., 2009; Matukumalli et al., 2009; Hayes, 2011; Heaton et al., 2014). Currently, DNA-based parentage analysis is shifting from the use of microsatellite (MS) to SNP markers. A panel of SNP has been recently adopted by the International Committee of Animal Recording (http://www.icar.org/) and by the International Society for Animal Genetics (ISAG, http://www.isag.us/) for cattle parentage testing (http://www.isag.us/Docs/ Cattle-SNP-ISAG-core-additional-panel-2013.xlsx).

Other comparison ring tests for parentage with a core panel of 100 SNP plus an additional panel of 100 SNP have recently been undertaken in cattle (Strucken et al., 2014). A panel of 163 SNP has also been proposed for parentage testing of sheep (Heaton et al., 2014).

The use of molecular markers for parentage analysis has been accompanied by the development of several statistical techniques for data management, principally based on exclusion, categorical, or fractional allocation and full probability (Jones et al., 2010).

A goat 53K SNP array has been recently developed by Illumina (San Diego, CA), in collaboration with the 
International Goat Genome Consortium (http://www. goatgenome.org/; Tosser-Klopp et al., 2014). In spite of the high potential of such a tool in goat breeding, a few technical issues still partially hamper its full exploitation. One of the main issues concerns the early stage of the reference goat genome assembly (Dong et al., 2013). One of the main parameters used to select SNP for parentage assessment (PA) in other species (e.g., cattle) is physical distance and linkage disequilibrium (LD) among markers (Strucken et al., 2014). Although recent studies have characterized overall LD in some goat breeds (Brito et al., 2015), inaccurate SNP positioning on the goat genome makes traditional methods based on physical distance less effective for PA purposes (Benjelloun et al., 2015; Bickhart et al., 2015).

This paper evaluates a new 3 -step procedure to select a small number of SNP for PA in goat. Advantages of this procedure are particularly relevant for species without an accurate SNP positioning, such as the goat. Our selection procedure of informative SNP markers is independent from SNP positioning, and it is based on parent-offspring identification by assessment of Mendelian errors (MDE), canonical discriminant analysis, and backward stepwise regression.

At present, the official MS-based method for parentage analysis in goats is generally too expensive for this species, considering the limited economic value of a single animal (Strucken et al., 2014). As already observed in sheep, the introduction of low-density SNP panels coupled with a cost-effective DNA-based technique would allow a $40 \%$ reduction of the analysis cost compared with MS (Heaton et al., 2014). This decrease in price would likely increase the use of DNA-based parentage determination in goats and help overcome the major constraints in control of inbreeding and implementing genetic improvement strategies.

\section{MATERIALS AND METHODS}

\section{Animal Sampling and Genotyping}

Blood samples were collected from 154 animals belonging to the Alpine $(\mathrm{n}=109)$ and the Saanen $(\mathrm{n}=$ 22) goat breeds, reared in 3 flocks in Northern Italy, and the Teramana goat breed $(\mathrm{n}=23)$, reared in one flock in central Italy. Samples were collected according to the recommendations of the European Council (Council of Europe, 1986) on animal care. The DNA was extracted from whole blood using a commercial kit (NucleoSpin Blood, Macherey-Nagel, Düren, Germany) following the manufacturer's instructions. The DNA samples were genotyped using the Illumina GoatSNP50 BeadChip (Tosser-Klopp et al., 2014).
All Alpine goats (8 males and 101 females) were used as the reference data set (REF), whereas 22 Saanen females (VAL1) and 23 (2 males, 21 females) Teramana individuals (VAL2) were used as the validation data set.

According to pedigree data, 46 out of 109 individuals in REF formed 20 trios (father, mother, and offspring), and in total 58 animals belonged to 50 parent-offspring (PO) pairs.

Group VAL1 included 5 PO pairs. Group VAL2 included one trio and 5 PO pairs. Group VAL2 animals were from the only existing flock of Teramana, an endangered breed consisting of about 80 animals recorded by the National Breeder Association (http:// www.assonapa.com/) that was chosen specifically for its small size and difficult PA. The latter is due to high inbreeding, which increases the number of cryptic relationships, leading to PO classification instead of full-sib.

To confirm our results, 53 animals belonging to all trios in the REF data set were also analyzed with the official MS parentage test (13 different markers: $H S C$, ILSTS19, INRA005, INRA063, MAF65, SRCRSP5, SRCRSP8, SRCRSP24, ILSTS23, INRA023, MCM527, CSRD247, SRCRSP23). This test was performed in outsourcing at the official Laboratory of the Italian Breeders Association (AIA-LGS, http://www.lgscr.it/ it/chi.htm).

\section{Data Set Preparation}

The REF genotype data were quality checked according to the following thresholds: SNP call rate $\geq 0.95$; minor allele frequency (MAF) $\geq 0.01$; individual genotype call rate $\geq 0.90$; and in Hardy-Weinberg equilibrium (Bonferroni corrected threshold, $P \leq 1 \times 10^{-7}$ ). The resulting data set was used in the initial genetic population analysis and for PA. A characterization of the animals included in the REF data set was performed by PLINK 1.07 Multidimensional Scaling (Purcell et al., 2007) to verify the absence of sampling errors. For the selection of a low-density SNP panel, all markers with MAF $\leq 0.3$, unknown chromosomal assignment or placement on the sexual chromosomes were excluded.

\section{SNP Selection and PA}

The 3-step procedure to select candidate SNP for the PA panel consisted of (1) the identification of real PO by MDE, (2) the identification of informative SNP by canonical discriminant analysis, and (3) reduction of the number of markers by MDE and stepwise regression. 
Mendelian error was calculated using an in-house script. Mendelian error is a simple comparison among the genotypes of 2 animals, checking for incompatible homozygosity. A Mendelian error occurs when opposing homozygotes are present at the same locus (e.g., AA for the sire and BB for the offspring). Although MDE for 2 PO pairs should be 0 on a $50 \mathrm{~K}$ panel, a low number of MDE are allowed to account for genotyping errors (Hayes et al., 2009). Pairwise comparisons were performed among all 109 REF animals, for a total of 5,886 comparisons. Each pair of individuals was classified as PO or not PO (NPO) based on MDE. Considering the SNP that remained after quality control check (MAF $\geq 1 \%$, SNP call rate $\geq 95 \%$, individual call rate $\geq 90 \%$, and not in Hardy-Weinberg equilibrium), we classified as PO all pairs with $<1,000 \mathrm{MDE}$ (Hayes et al., 2009).

A canonical discriminant analysis (CANDISC), included in the R package MASS (Venables and Ripley, 2002), was then performed on the REF data set. A CANDISC was applied as a dimensionality reduction method that uses a linear combination of variables to separate 2 or more known groups. To characterize each group, the method gives a linear score to each variable (i.e., in our case markers) to best assign each observation to its group (Fisher, 1936). We performed CANDISC one chromosome at a time to keep the number of markers lower than the number of pairwise comparisons. A CANDISC was performed using pairwise individuals' genotype comparison as predictive variables and $\mathrm{PO}-$ NPO classification as discriminating groups. The SNP with extreme discriminant linear scores (mean $\pm 2 \mathrm{SD}$ ) were retained, whereas all redundant markers $(|\mathrm{r}|>0.7$ in pairwise comparisons) were discarded. It is worth noting that, although no direct LD measure was used in this work, the exclusion of redundant markers indirectly accounts for local LD among markers. We then reduced the panel size to 200 SNP, retaining only the markers that showed the highest number of MDE in NPO group and lowest in the PO group. This size was chosen according to the ISAG panel for PA in cattle (http:// www.isag.us/Docs/Cattle-SNP-ISAG-core-additionalpanel-2013.xlsx) and is an intermediate step before final reduction. Finally, we further reduced the panel size using backward stepwise regression, excluding one marker at a time by using MDE as response vector, until we found the lowest number of variables able to identify groups with $100 \%$ specificity. According to ISAG standards, parentage assignment was performed by MDE estimation on the reduced SNP panel. For each identified panel, we also calculated the following performance parameters: sensitivity, specificity, accuracy, median estimates for probability of a coincidental match between 2 animals (Heaton et al., 2014), and the fraction of potential adults excluded from parent- age test (Heaton et al., 2014). Sensitivity is the ratio of true positives identified by the test on the total number of true parents; specificity is the ratio of nonparents excluded by the test to the total number of nonparents; and accuracy is the ratio of correct assignment of the test to the total number of tests performed. These parameters can range from 0 to 1 for the worst and best scenario, respectively. The probability of a random coincidental match at an SNP locus between random animals is $\boldsymbol{P}_{\mathrm{i}}$. The $P_{\mathrm{i}}$ for locus A with SNP alleles A1 and A2 was the sum of the squares of the 3 genotype frequencies. Values were calculated with the following formula: $P_{\mathrm{i}}=\left(f_{\mathrm{A} 1 \mathrm{~A} 1}\right)^{2}+\left(f_{\mathrm{A} 1 \mathrm{~A} 2}\right)^{2}+\left(f_{\mathrm{A} 2 \mathrm{~A} 2}\right)^{2}$, where $f_{\mathrm{A} 1 \mathrm{~A} 1}$, $f_{\mathrm{A} 1 \mathrm{~A} 2}$, and $f_{\mathrm{A} 2 \mathrm{~A} 2}$ are the relative genotypic frequencies of $\mathrm{A}_{1} \mathrm{~A}_{1}, \mathrm{~A}_{1} \mathrm{~A}_{2}$, and $\mathrm{A}_{2} \mathrm{~A}_{2}$, respectively. The combined $P_{\mathrm{i}}$ for multiple SNP markers is the product of the $P_{\mathrm{i}}$ values of individual markers. The probability of observing opposing homozygotes at a SNP locus between a random eligible adult and a random offspring is $\boldsymbol{P}_{\mathbf{e}}$. Values for biallelic loci and with information on only one parent were calculated using the formula $P_{\mathrm{e}}=2 \times\left(f_{\mathrm{A} 1 \mathrm{~A} 1} \times\right.$ $f_{\mathrm{A} 2 \mathrm{~A} 2}$ ), where $f_{\mathrm{A} 1 \mathrm{~A} 1}$ and $f_{\mathrm{A} 2 \mathrm{~A} 2}$ are the relative genotype frequencies of $\mathrm{A}_{1} \mathrm{~A}_{1}$ and $\mathrm{A}_{2} \mathrm{~A}_{2}$, respectively. Combined $P_{\mathrm{e}}$ for multiple SNP was calculated as follows: $P_{\mathrm{e}(\mathrm{SNP} n)}$ $=R_{0} \times P_{\mathrm{e}(\mathrm{SNP} 1)}+R_{1} \times P_{\mathrm{e}(\mathrm{SNP} 2)}+R_{2} \times P_{\mathrm{e}(\mathrm{SNP} 3)} \cdots$ $+R_{n-1} \times P_{\mathrm{e}(\mathrm{SNP} n)}$, where $P_{\mathrm{e}(\mathrm{SNP} 1)}$ was the fraction of eligible adults excluded by the first SNP and $R$ was the remaining fraction of unexcluded adults $\left[R_{0}=1, R_{1}=\right.$ $R_{n-1}-\left(R_{n-1} \times P_{\mathrm{e}}\right)=R_{0}-\left(R_{0} \times P_{\mathrm{e}}\right), R_{2}=R_{1}-\left(R_{1}\right.$ $\times P_{\mathrm{e}}$ ), and so on]. To improve data clearness, we report the fraction of potential adults excluded $\left(P_{\mathrm{e}}\right)$ per single SNP as a fraction of the potential adults retained (PR; $\mathrm{PR}=1-P_{\mathrm{e}}$ ) from the parentage test.

\section{RESULTS AND DISCUSSION}

\section{PA on the Whole 50K SNP Panel}

After the preliminary editing, a total of 49,609 SNP were retained in the REF data set.

A multidimensional scaling plot was produced to verify the absence of population substructure. The analysis did not reveal any strong population structure, except for a mild sire effect (Figure 1).

Mendelian errors confirmed 10 pedigree-based trios out of 20 , resulting in a $50 \%$ trio pedigree error rate (Table 1). In addition, 5 new undeclared trios were identified, increasing the total number of related animals. All trios identified by the above analyses were confirmed by the official MS parentage test. Interestingly, MDE failed to identify PO pair of individuals identified by MS. This pair of individuals (a buck and its putative offspring) did not pass the MDE threshold with the 50K SNP panel, yielding 1,479 MDE. Men- 
Table 1. Pedigree information and SNP identification for the reference (REF) and the validation (VAL1 and VAL2) data sets ${ }^{1}$

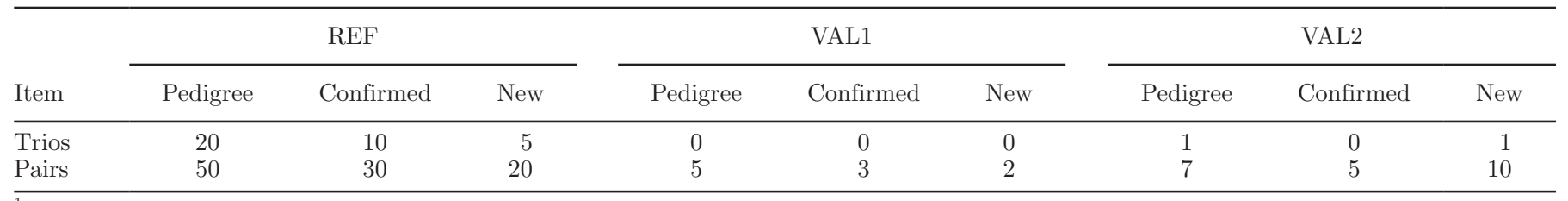

${ }^{1}$ For each data set, we reported the number of pedigree-based trios and pairs, the number of trios and pairs confirmed by SNP, and the number of new trios and pairs identified by SNP.

delian error distributions for pairwise comparisons are reported on Supplemental Figure S1 (http://dx.doi. org/10.3168/jds.2015-10077). In conclusion, our analysis confirmed 30 out of 50 declared pedigree PO (error rate $=40 \%$ ). However, PA identified 20 more pairs of $\mathrm{PO}$, for a total of $50 \mathrm{PO}$ pairs.

\section{Parentage Analysis with 200 and 130 SNP Panels}

After PA, a second and more stringent editing was applied on the REF data set (MAF $>0.3$, no markers with unknown position or on heterochromosomes). A total of 27,523 SNP were retained for further analyses.

As described above, only an indirect marker exclusion by LD was applied, because of the uncertain positioning of the SNP loci on the reference genome map that could heavily affect the results. In fact, direct LD estimation on 36 not closely related individuals (MDE $\geq 2,020$ ) showed a strong smear, composed of markers with very high level of $\mathrm{r}^{2}$ at high distances (1-10 Mb, Figure $2 \mathrm{a}$ and $2 \mathrm{~b}$ ) and SNP very close with low $\mathrm{r}^{2}$ values (Figure 2).

A CANDISC reduction identified 1,206 highly polymorphic SNP markers, which were further thinned to 1,133 after the exclusion of collinear SNP.

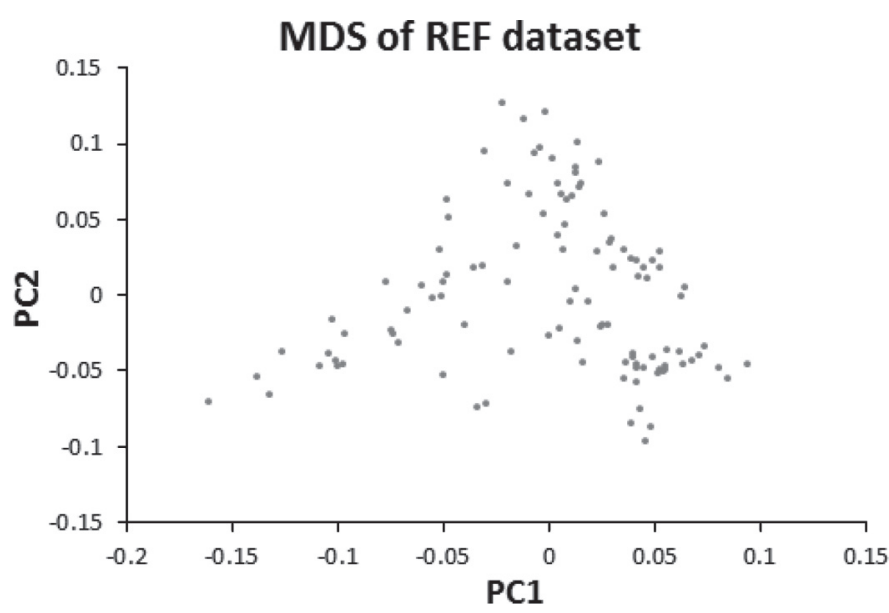

Figure 1. Multidimensional scaling (MDS) plot of the reference (REF) panel, showing absence of relevant substructures among animals. $\mathrm{PC}=$ principal component. a)

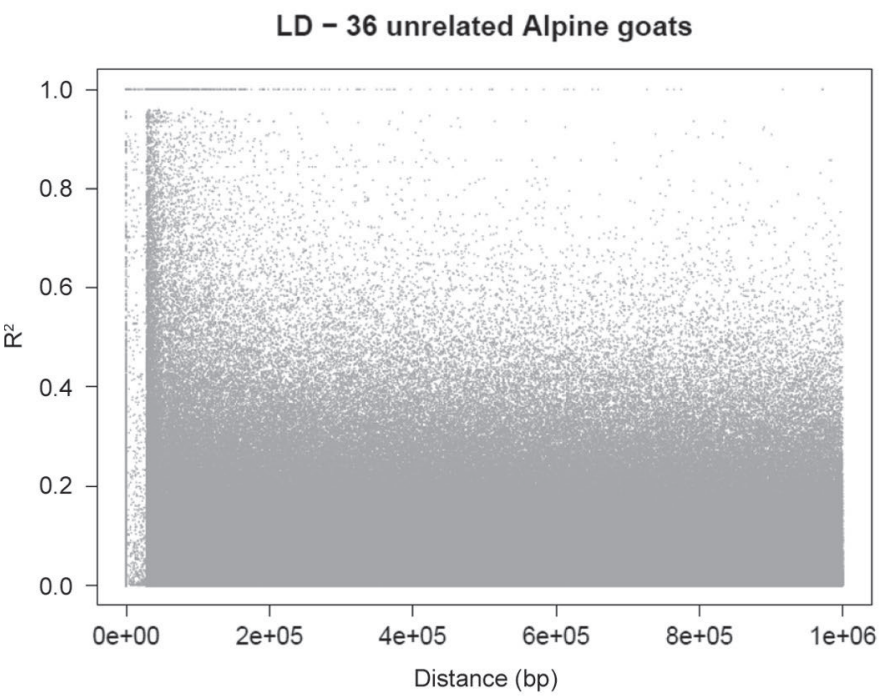

b)

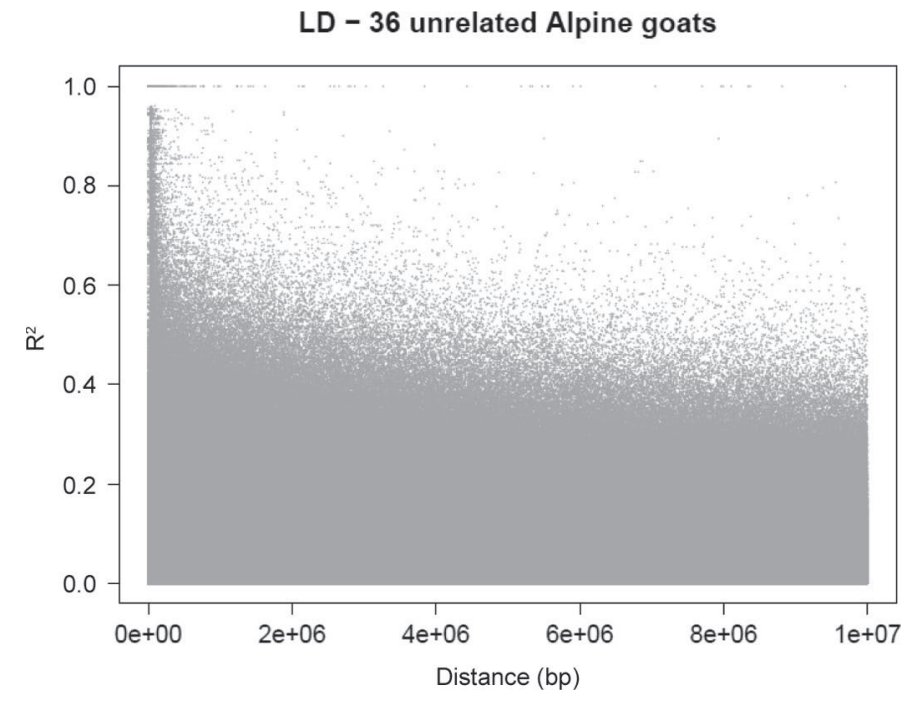

Figure 2. Linkage disequilibrium (LD) plot for our data, estimated using PLINK 1.07 software (Purcell S., Boston, MA). On the x-axis, we report the distance in base pairs, and on the y-axis we report the LD level estimated as $r^{2}$. The plot shows smearing at (a) distances lower than $1 \mathrm{Mb}$ and (b) distances lower than $10 \mathrm{Mb}$. In addition, (a) markers shows anomalous $\mathrm{r}^{2}$ values at very close distances, as shown. 
The 200 SNP panel was obtained by identifying the markers with the highest MDE in the NPO group and the lowest MDE in the PO group (Supplemental Table S1; http://dx.doi.org/10.3168/jds.2015-10077). We chose this panel size not because it showed the best performance, but because this is the number of markers in the ISAG standard panel for PA in cattle. The thinning procedure led to an uneven distribution of the selected markers on the genome, independent from differences in marker density and length of chromosomes (Figure 3a).

We checked the assessment power of the 200 SNP data set according to ISAG standards (http://www. isag.us/docs/guideline-for-cattle-snp-use-for-parent-

a)

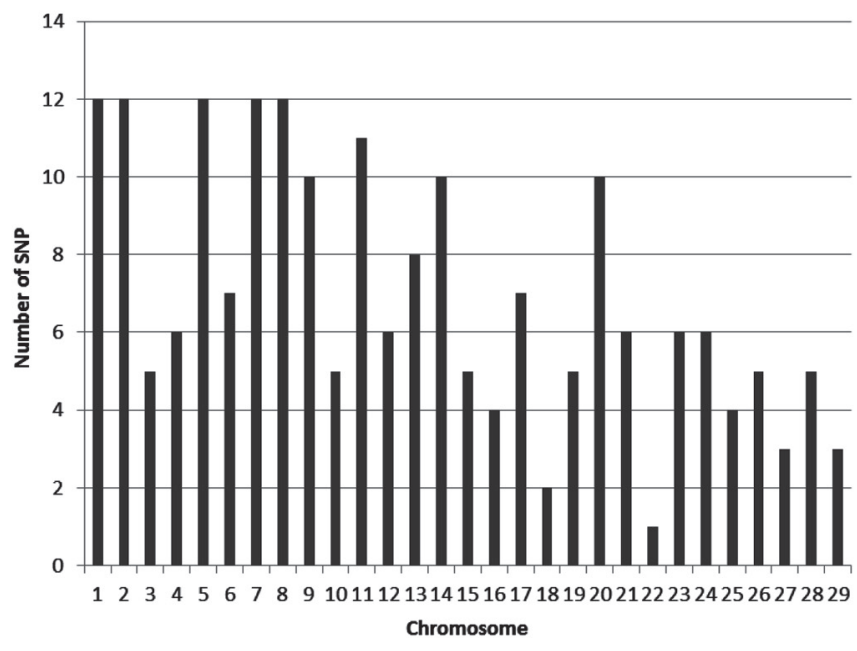

b)

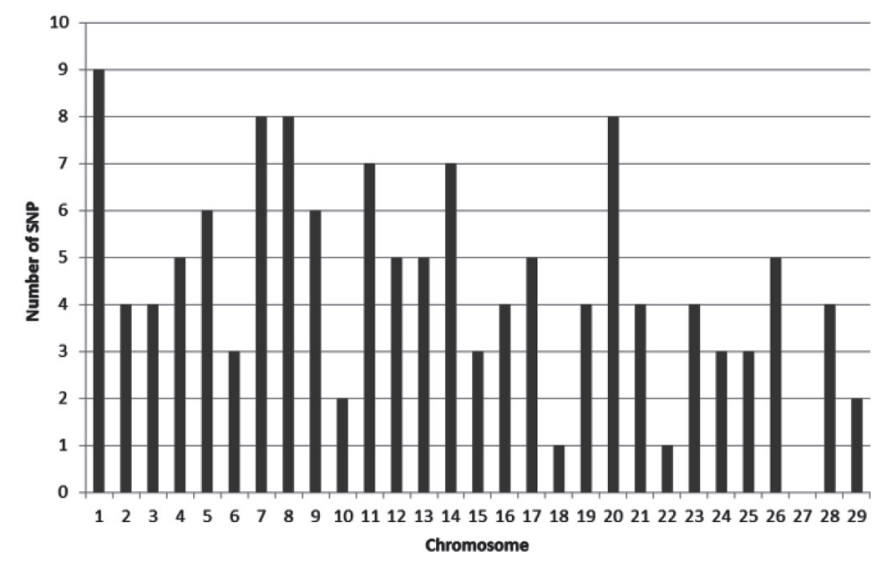

Figure 3. Chromosomal distribution of (a) 200 and (b) 130 identified SNP. (a) All chromosomes have at least one marker in the 200 SNP panel. (b) All chromosomes but one (27) have at least one marker in the 130 SNP panel. age-2012.pdf). Parentage was assigned as PO in the case of 0 to $1 \mathrm{MDE}$, unassigned and doubtful in the case of 2 to $3 \mathrm{MDE}$, and unassigned in the case of more than 3 MDE. The 200 SNP panel tested on the REF population had $100 \%$ sensitivity, specificity, accuracy; low PR; and low $P_{\mathrm{i}}$, as reported in Table 2. Mean of single SNP $P_{\mathrm{e}} \pm$ standard deviation (SD) was $0.1505 \pm 0.0133\left(P_{\mathrm{e}}\right.$ values per SNP are reported in Supplemental Table S2; http://dx.doi.org/10.3168/ jds.2015-10077). As expected, the exclusion power of a single SNP is far lower than for a single MS (up to 0.5; Bolormaa et al., 2008), because of the higher variability of the second compared with the first (SNP are only biallelic, whereas MS are multiallelic). On average, 2 or 3 SNP are needed per MS marker to obtain equivalent cumulative exclusion power (Al-Atiyat, 2015).

The panel was further reduced to 130 SNP by applying a backward stepwise regression on the previously identified 200 SNP on the REF data set (Supplemental Table S1; http://dx.doi.org/10.3168/jds.2015-10077). The distribution of the 130 markers on chromosomes is reported in Figure 3b. The reduction process led to an unequal distribution of markers on each chromosome, and all but one (chromosome 27) were represented in the panel.

The 130 SNP panel performed similarly to the 200 SNP panel with REF, having $100 \%$ sensitivity, specificity, and accuracy but slightly larger $\mathrm{PR}$ and $P_{\mathrm{i}}$ (as shown in Table 2). Mean of single SNP $P_{\mathrm{e}} \pm \mathrm{SD}$ was $0.1509 \pm 0.0142$, slightly larger than in the $200 \mathrm{SNP}$ panel $\left(P_{\mathrm{e}}\right.$ values per SNP are reported in Supplemental Table S2; http://dx.doi.org/10.3168/jds.2015-10077). The reduction in $P_{\mathrm{e}}$ and increase in $P_{\mathrm{i}}$ are due to the lower number of markers, which reduces the combined probability of exclusion and increases the probability of inclusion. Also, the increase in mean $P_{\mathrm{e}}$ per SNP was expected, because of the selection of more discriminant markers. For 49,609 SNP panels, mean of single SNP $P_{\mathrm{e}}$ $\pm \mathrm{SD}$ was $0.0886 \pm 0.0437$. These values are far lower than values for the low-density panels, supporting the efficacy of this markers selection method.

\section{Validation of the 200 and 130 SNP Panels}

Evaluating the power of the SNP panel for PA on the same data set used for SNP selection would likely lead to an overestimate of the performance. Thus, the 2 reduced panels were tested for PA in 2 validation data sets of different breeds, Saanen and Teramana (VAL1 and VAL2, respectively). Because MS and SNP-based PA were in close agreement on the REF population, VAL1 and VAL2 were analyzed using SNP data only.

Using the full panel in VAL1, 3 pairs of related animals were confirmed and 2 undeclared pairs were 


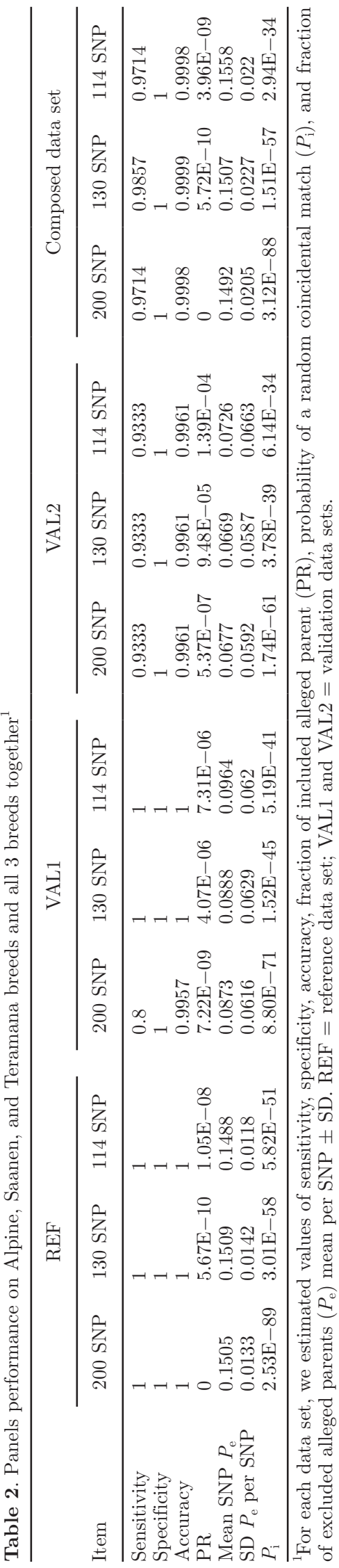

identified. In total, 5 pairs of related animals with low MDE were detected (Table 1). In VAL2, only one declared trio was excluded by MDE. In contrast, a total of 15 PO undeclared relationships were found. The 200 SNP panel showed high performance on VAL1, with no false positives and high values for specificity and accuracy. Only the sensitivity value was lower due to an undeclared pair of animals sharing 799 MDE. This pair of animals had borderline values for our less stringent MDE threshold of 1,000, thus we defined it as a PO pair. However, it is interesting to observe that the MDE threshold used in this study is to be considered too lenient for real case scenarios. In fact, using a more realistic threshold of $0.5 \%(\sim 250 \mathrm{MDE})$, which is still high, we would have classified this couple as NPO, and sensitivity and accuracy of the panel would have risen to $100 \%$. Therefore, the results presented here can be considered as an underestimate of the real potential of this method. Information given by pedigree registration indicated that these 2 animals were full-sibs. This kind of relationship is very difficult to diagnose by molecular markers, and prior information is useful to obtain a more accurate assignment. In fact, when pedigree reliability is low, full-sibs remain difficult to distinguish from PO. Both PR and $P_{\mathrm{i}}$ were low as shown in Table 2 . The average $P_{\mathrm{e}} \pm \mathrm{SD}$ per SNP was $0.0873 \pm 0.0616$ $\left(P_{\mathrm{e}}\right.$ values per SNP are reported in Supplemental Table S2; http://dx.doi.org/10.3168/jds.2015-10077). The same panel on VAL2 showed higher performances, with high values of specificity and accuracy and low values of PR and $P_{\mathrm{i}}$ as shown in Table 2. Only sensitivity was lower due to a false negative with MDE 920. As for VAL1, this pair was classified as PO because of the high MDE threshold. Again, the adoption of the more realistic threshold of $250 \mathrm{MDE}$ would have classified it as NPO. Also, in this case, sensitivity and accuracy would have increased to $100 \%$. Average $P_{\mathrm{e}} \pm$ $\mathrm{SD}$ per SNP was $0.0677 \pm 0.0592\left(P_{\mathrm{e}}\right.$ values per SNP are reported in Supplemental Table S2; http://dx.doi. org/10.3168/jds.2015-10077).

The 130 SNP panel performed differently in VAL1, still showing no false positives and 100\% assignment, when no prior information was considered. In any case, the panel showed a decreased PR and increased $P_{\mathrm{i}}$ when compared with the 200 SNP panel, as shown in Table 2. Mean $P_{\mathrm{e}}$ per $\mathrm{SNP}$ was $0.0888 \pm 0.0629\left(P_{\mathrm{e}}\right.$ values per SNP are reported in Supplemental Table S2; http:// dx.doi.org/10.3168/jds.2015-10077).

In VAL2, the 130 SNP panel performed slightly poorer compared with the 200 SNP panel. Sensitivity, specificity, and accuracy remained the same, still showing no false positives. However, also in this case, performance rose when a more realistic threshold to identify PO was used, showing perfect sensitivity, specificity, 
and accuracy. In both cases, also when considering the high MDE threshold, PR and $P_{\mathrm{i}}$ increased, as shown in Table 2. Mean single SNP $P_{\mathrm{e}} \pm \mathrm{SD}$ was $0.0669 \pm 0.0587$ $\left(P_{\mathrm{e}}\right.$ values per SNP are reported in Supplemental Table S2; http://dx.doi.org/10.3168/jds.2015-10077). Even though SNP showed a similar mean value on VAL1 and VAL2, the difference in their performance may be due to a different exclusion power of each marker. Each marker has different $P_{\mathrm{e}}$ values in the 2 data sets, with some SNP having no exclusion power in 1 of the 2 breeds. High variability of $P_{\mathrm{e}}$ values among breeds is also due to different allelic frequencies of each SNP among populations, which could negatively affect efficacy of the test. This further underlines the need to include more breeds in the discovery phase, to assess the performance of the panel on a larger set of breeds.

\section{Final Reduction to 114 SNP Panel}

A third reduced panel was constructed performing stepwise regression on the 200 SNP panel that included animals from REF, VAL1, and VAL2 data sets, for a total of 11,781 pairwise comparisons among individuals. The reduction process led to the identification of a 114 SNP panel (chromosomal distribution on Figure 4). These markers were distributed on all but 2 chromosomes (chromosomes 26 and 27) and are reported in Supplemental Table S1 (http://dx.doi.org/10.3168/ jds.2015-10077). This panel showed high sensitivity, specificity, and accuracy and low $\mathrm{PR}$ and $P_{\mathrm{i}}$, as shown in Table 2. As in the previous panels, a more realistic MDE threshold to identify PO resulted in a panel performance matching all true PO pairs and excluding all NPO pairs. Mean single SNP $P_{\mathrm{e}} \pm \mathrm{SD}$ was $0.1558 \pm$ $0.0220\left(P_{\mathrm{e}}\right.$ values per SNP are reported in Supplemental Table S2; http://dx.doi.org/10.3168/jds.2015-10077). Overall performance of this panel was comparable to the 2 larger panels.

\section{Random Selection of Markers}

To evaluate the efficacy of our 3-step method, 100 random data sets for each of the panel subsets $(200,130$, and 114 SNP) were randomly extracted from 27,523 highly polymorphic SNP in Alpine breeds. Each random panel was tested on a data set including within- and across-breeds pairwise comparison, for a total of 11,781 pairs of animals. The 100 randomly chosen panels of comparable size for the 200 SNP panel were equivalent to our method in $66 \%$ of cases. However, when reducing the number of SNP to 130 and 114, the random choice of markers showed comparable results only on 2 and $0 \%$ of the cases, respectively (Supplemental Table S3; http://dx.doi.org/10.3168/jds.2015-10077).
Moreover, we also evaluated our random panels on 10,338 highly polymorphic SNP in all 3 breeds (MAF $>0.3$ in each breed). Again, the 100 randomly chosen panels of comparable size for the 200 SNP panel were comparable to our method in $81 \%$ of cases. However, when reducing the number of SNP to 130 and 114, the random choice of markers showed comparable results only in 9 and $0 \%$ of the cases, respectively (Supplemental Table S3; http://dx.doi.org/10.3168/jds.201510077).

These results, if confirmed when using a wider set of breeds in the reference population, suggest that the 200 SNP panel chosen to match the bovine ISAG SNP panel size is not the best choice in terms of PA accuracy on goats. In addition, our results would confirm that our method is able to efficiently identify small SNP panels for PA and that MAF-based selection alone is ineffective for identification of useful PA panels.

\section{CONCLUSIONS}

These results suggest that the proposed method can successfully identify a reduced number of markers able to assess parentage in species with low map resolution. The identification of small panels can easily help in assessing direct relationships among animals, enhancing the control of inbreeding and the effectiveness of genetic improvement programs. In spite of the promising results obtained, it is necessary to assess the proposed procedure independently in a wider set of breeds, to identify the smallest panel for PA in different goat breeds. This study is a first step toward the implementation of new technologies for PA in species with low genomic information, such as the goat. Such species,

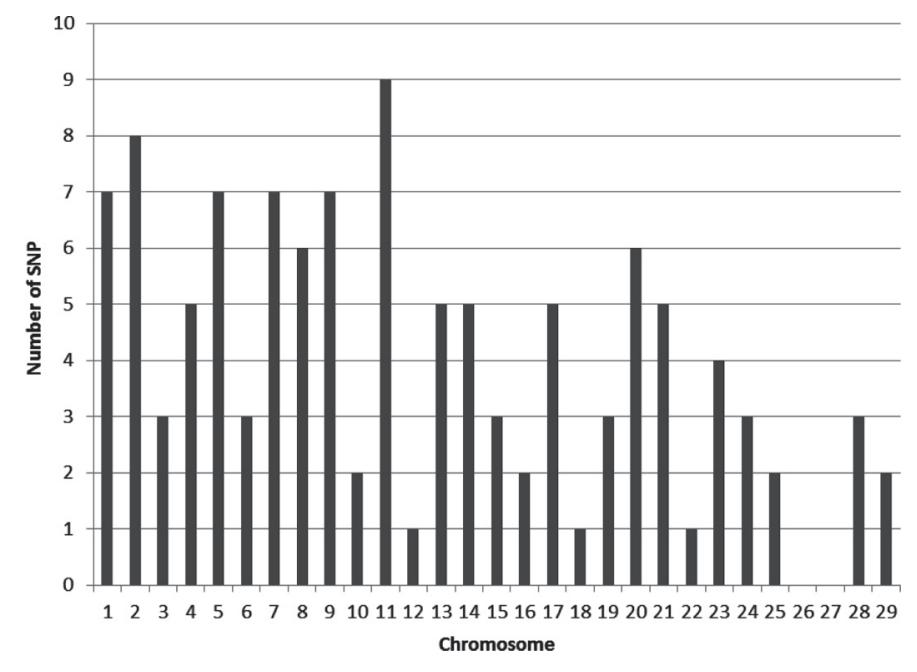

Figure 4. Chromosomal distribution of 114 identified SNP. All chromosomes but 2 (26 and 27) have at least one marker in the panel. 
which are often economically relevant in marginal, rural areas of the world, would greatly benefit from the availability of low-cost tools for breeding management and genetic improvement.

\section{ACKNOWLEDGMENTS}

This research received funding by project "Innovagen" funded by the Italian Ministry of Agriculture (MiPAAF, Rome, Italy). Talenti and Frattini were supported by the Doctoral School on Veterinary and Animal Sciences of the Veterinary Faculty of the University of Milan (Italy). Coizet and Nicoloso were supported by GenHome Project (Lodi, Italy).

\section{REFERENCES}

Al-Atiyat, R. M. 2015. The power of 28 microsatellite markers for parentage testing in sheep. Electron. J. Biotechnol. 18:116-121. http://dx.doi.org/10.1016/j.ejbt.2015.01.001.

Benjelloun, B., F. J. Alberto, I. Streeter, F. Boyer, E. Coissac, S. Stucki, M. BenBati, M. Ibnelbachyr, M. Chentouf, A. Bechchari, K. Leempoel, A. Alberti, S. Engelen, A. Chikhi, L. Clarke, P. Flicek, S. Joost, P. Taberlet, and F. Pompanon. 2015. Characterizing neutral genomic diversity and selection signatures in indigenous populations of Moroccan goats (Capra hircus) using WGS data. Front. Genet. 6:107. http://dx.doi.org/10.3389/fgene.2015.00107.

Bickhart, D., S. Koren, A. Phyllippy, T. Smith, J. Burton, I. Liachko, T. Sonstegard, C. Van Tassell, H. Huson, and S. Schroeder. 2015. The Use of PacBio and Hi-C Data in Denovo Assembly of the Goat Genome. Plant Anim. Genome, San Diego, CA.

Bolormaa, S., A. Ruvinsky, S. Walkden-Brown, and J. van der Werf. 2008. DNA-based parentage verification in two Australian goat herds. Small Rumin. Res. 80:95-100. http://dx.doi.org/10.1016/j. smallrumres.2008.08.005.

Brito, L. F., M. Jafarikia, D. A. Grossi, J. W. Kijas, L. R. Porto-Neto, R. V. Ventura, M. Salgorzaei, and F. S. Schenkel. 2015. Characterization of linkage disequilibrium, consistency of gametic phase and admixture in Australian and Canadian goats. BMC Genet. 16:67. http://dx.doi.org/10.1186/s12863-015-0220-1.

Council of Europe. 1986. European Convention for the Protection of Vertebrate Animals Used for Experimental and Other Scientific Purposes. CETS 123. Counc. Eur., Strasbourg, France.

Dong, Y., M. Xie, Y. Jiang, N. Xiao, X. Du, W. Zhang, G. TosserKlopp, J. Wang, S. Yang, J. Liang, W. Chen, J. Chen, P. Zeng, Y. Hou, C. Bian, S. Pan, Y. Li, X. Liu, W. Wang, B. Servin, B. Sayre, B. Zhu, D. Sweeney, R. Moore, W. Nie, Y. Shen, R. Zhao, G. Zhang, J. Li, T. Faraut, J. Womack, Y. Zhang, J. Kijas, N. Cockett, X. Xu, S. Zhao, J. Wang, and W. Wang. 2013. Sequencing and automated whole-genome optical mapping of the genome of a domestic goat (Capra hircus). Nat. Biotechnol. 31:135-141. http://dx.doi.org/10.1038/nbt.2478.

Fisher, P. J., B. Malthus, M. C. Walker, G. Corbett, and R. J. Spelman. 2009. The number of single nucleotide polymorphisms and on-farm data required for whole-herd parentage testing in dairy cattle herds. J. Dairy Sci. 92:369-374. http://dx.doi.org/10.3168/ jds.2008-1086.

Fisher, R. A. 1936. The use of multiple measurements in taxonomic problems. Ann. Eugen. 7:179-188. http://dx.doi. org/10.1111/j.1469-1809.1936.tb02137.x.

Hayes, B. J. 2011. Technical note: Efficient parentage assignment and pedigree reconstruction with dense single nucleotide polymorphism data. J. Dairy Sci. 94:2114-2117. http://dx.doi.org/10.3168/ jds.2010-3896.

Hayes, B. J., P. J. Bowman, A. C. Chamberlain, K. Verbyla, and M. E. Goddard. 2009. Accuracy of genomic breeding values in multibreed dairy cattle populations. Genet. Sel. Evol. 41:51. http:// dx.doi.org/10.1186/1297-9686-41-51.

Heaton, M. P., G. P. Harhay, G. L. Bennett, R. T. Stone, W. M. Grosse, E. Casas, J. W. Keele, T. P. L. Smith, C. G. Chitko-McKown, and W. W. Laegreid. 2002. Selection and use of SNP markers for animal identification and paternity analysis in U.S. beef cattle. Mamm. Genome 13:272-281. http://dx.doi.org/10.1007/s00335001-2146-3.

Heaton, M. P., K. a. Leymaster, T. S. Kalbfleisch, J. W. Kijas, S. M. Clarke, J. McEwan, J. F. Maddox, V. Basnayake, D. T. Petrik, B. Simpson, T. P. L. Smith, and C. G. Chitko-McKown. 2014. SNPs for parentage testing and traceability in globally diverse breeds of sheep. PLoS ONE 9:e94851. http://dx.doi.org/10.1371/journal. pone.0094851.

Jones, A. G., C. M. Small, K. a. Paczolt, and N. L. Ratterman. 2010. A practical guide to methods of parentage analysis. Mol. Ecol. Resour. 10:6-30. http://dx.doi.org/10.1111/j.1755-0998.2009.02778.x.

Matukumalli, L. K., C. T. Lawley, R. D. Schnabel, J. F. Taylor, M. F. Allan, M. P. Heaton, J. O'Connell, S. S. Moore, T. P. L. Smith, T. S. Sonstegard, and C. P. Van Tassell. 2009. Development and characterization of a high density SNP genotyping assay for cattle. PLoS ONE 4:e5350. http://dx.doi.org/10.1371/journal. pone.0005350.

Purcell, S., B. Neale, K. Todd-Brown, L. Thomas, M. R. Ferreira, D. Bender, J. Maller, P. Sklar, P. I. W. de Bakker, M. J. Daly, and P. C. Sham. 2007. PLINK: A tool set for whole-genome association and population-based linkage analyses. Am. J. Hum. Genet. 81:559-575. http://dx.doi.org/10.1086/519795.

Strucken, E. M., B. Gudex, M. H. Ferdosi, H. K. Lee, K. D. Song, J. P. Gibson, M. Kelly, E. K. Piper, L. R. Porto-Neto, S. H. Lee, and C. Gondro. 2014. Performance of different SNP panels for parentage testing in two East Asian cattle breeds. Anim. Genet. 45:572-575. http://dx.doi.org/10.1111/age.12154.

Tosser-Klopp, G., P. Bardou, O. Bouchez, C. Cabau, R. Crooijmans, Y. Dong, C. Donnadieu-Tonon, A. Eggen, H. C. M. Heuven, S. Jamli, A. J. Jiken, C. Klopp, C. T. Lawley, J. McEwan, P. Martin, C. R. Moreno, P. Mulsant, I. Nabihoudine, E. Pailhoux, I. Palhière, R. Rupp, J. Sarry, B. L. Sayre, A. Tircazes, J. Wang, W. Wang, and W. Zhang.International Goat Genome Consortium. 2014. Design and characterization of a 52K SNP chip for goats. PLoS ONE 9:e86227. http://dx.doi.org/10.1371/journal.pone.0086227.

Venables, W. N., and B. D. Ripley. 2002. MASS: Modern Applied Statistics with S. Springer, New York, NY. http://dx.doi.org/10.1198/ tech.2003.s33.

Werner, F. A., G. Durstewitz, F. A. Habermann, G. Thaller, W. Krämer, S. Kollers, J. Buitkamp, M. Georges, G. Brem, J. Mosner, and R. Fries. 2004. Detection and characterization of SNPs useful for identity control and parentage testing in major European dairy breeds. Anim. Genet. 35:44-49. http://dx.doi.org/10.1046/j.13652052.2003.01071.x. 